\title{
Unités de soins au fort potentiel
}

\author{
Jolanda Contartese ${ }^{a}$, Werner Z'Graggen ${ }^{b}$, Brigitte Hämmerlic, Luca Lavinad \\ ${ }^{a}$ Dr méd., présidente de la CRUIMC, Hôpital cantonal de Baden; ' Prof. Dr méd., vice-président de la CRUIMC, Hôpital de I'lle de Berne; \\ ${ }^{c}$ Membre du Présidium Soins CRUIMC, Hôpital de I'lle de Berne; ' Secrétariat général SSMI, IMK SA, Bâle
}

Le premier symposium Swiss Intermediate Care s'est tenu le 24 janvier 2020 à l'Hôpital de l'Ile de Berne. Outre la pluralité des U-IMC, cette manifestation d'une journée a principalement porté sur les directives récemment révisées permettant aux unités de soins intermédiaires d'être reconnues en Suisse. Les défis éthiques et la facturation des prestations y ont également été abordés.

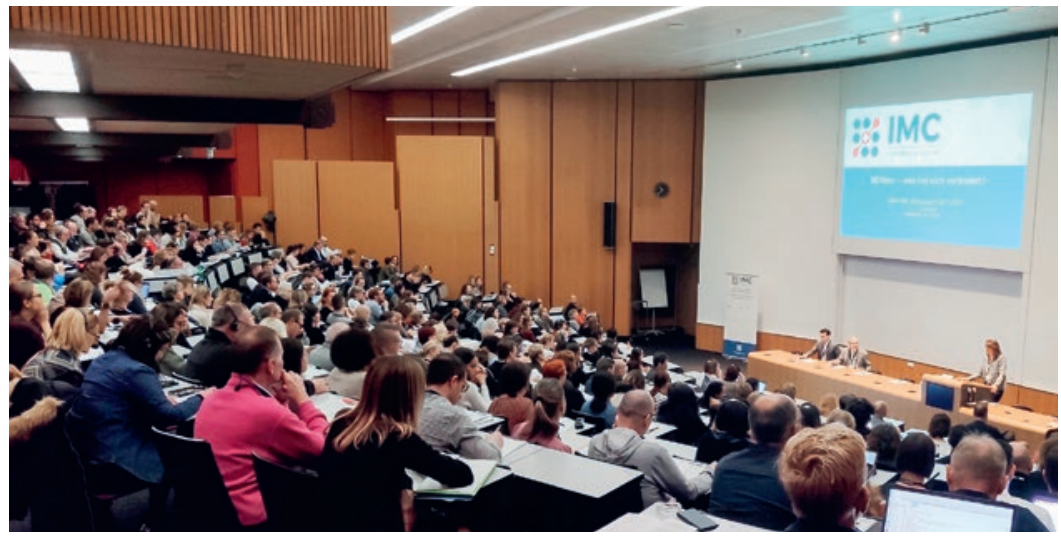

Lors du premier symposium Swiss Intermediate Care ont été évoquées les directives révisées relatives aux unités de soins intermédiaires.

Le premier symposium Swiss Intermediate Care a été organisé par la Commission de reconnaissance des unités de soins intermédiaires (CRUIMC), organe interdisciplinaire comptant près de 30 représentants issus de neuf sociétés spécialisées. Lors de cette journée, près de 300 personnes ont assisté à des exposés portant sur les différents aspects des soins intermédiaires ou pris part à des ateliers sur des thèmes tels que l'ECG ou la ventilation non invasive. Un immense succès qui souligne l'importance et la pertinence de ces services dans les soins de santé suisses, importance ayant, une fois encore, été démontrée au cours des dernières semaines et des derniers mois de la pandémie de COVID-19. En effet, avec les unités de soins intensifs, les U-IMC de Suisse ont probablement été les services hospitaliers les plus sollicités du pays.

\section{Directives révisées depuis janvier 2020}

Depuis 2016, la CRUIMC se rend dans les U-IMC des hôpitaux de toute la Suisse et procède à leur recon- naissance en s'appuyant sur les directives entrées en vigueur pour la première fois début 2014. Comme le rappelle le vice-président de la CRUIMC, Werner Z'Graggen de Berne, ces directives sont révisées tous les cinq ans par la CRUIMC et les sociétés spécialisées représentées et adaptées aux évolutions des unités de soins intermédiaires et de la médecine en général. La première révision des directives a ainsi eu lieu l'an dernier. Les directives actualisées ont été adoptées par les neuf sociétés spécialisées à l'automne dernier et sont en vigueur depuis janvier de cette année. Les trois principales modifications apportées aux directives concernent la procédure de reconnaissance, l'adaptation du nombre de journées de soins et la formation postgraduée des médecins responsables. En outre, les personnes nécessitant des soins en U-IMC sont désormais désignées comme "patients courant un risque vital latent». Cette définition faisait défaut jusqu'ici.

\section{Des unités de soins très diverses}

L'élaboration d'origine et la révision des directives intervenue l'an dernier se sont avérées compliquées en raison de la diversité des U-IMC de Suisse. Les différences existant entre ces services en Suisse ont pu être constatées clairement lors du symposium Intermediate Care grâce aux présentations axées sur la pratique des U-IMC qu'ils dirigent par les membres de la CRUIMC (Roland Darms de Bâle, Mattia Arrigo de Zurich, Emmanuel Carrera de Genève et Juan Llor de Sion). Certaines U-IMC interdisciplinaires sont spécialisées dans la prise en charge de tous les patients de l'hôpital dont l'état de santé ne nécessite pas de soins intensifs mais qui courent malgré tout, d'une manière ou d'une autre, un risque vital latent, alors que d'autres s'occupent exclusivement de patients présentant certains tableaux cliniques. Toutes les U-IMC ont néan- 
moins en commun de traiter des patients dont l'état de santé est relativement critique. Les autres facteurs sont en revanche très hétérogènes.

\section{Importance des directives anticipées}

Dans les unités de soins intensifs comme dans les U-IMC, les questions d'ordre éthique jouent un rôle très important en raison de l'état généralement critique des patients. La volonté présumée du patient est le point central dans presque toutes les questions éthiques, explique Rouven Porz de Berne. Les directives anticipées sont ici centrales, explique-t-il, car le patient y indique sa volonté pour le cas où il ne serait plus en capacité de porter un jugement. En dépit de l'importance majeure des directives anticipées du patient, très peu en disposent, même chez les patients très âgés, explique Martial Coutaz de Sion.

Même si les questions portant sur la volonté présumée du patient sont souvent difficiles à interpréter en dépit de directives anticipées, les vrais dilemmes sont rares dans le quotidien clinique, ajoute Rouven Porz. Le plus souvent, c'est le manque de communication au sein de l'équipe soignante qui soulève des questions prétendument éthiques. Il est donc essentiel de promouvoir une culture ouverte du dialogue permettant également la critique envers les supérieurs et les membres de l'équipe. Evelyne Binsack, guide de montagne, alpiniste, pilote d'hélicoptère et autrice, est allée encore plus loin dans sa présentation lors du symposium: elle recommande l'élaboration d'un plan qui serait défini de manière concrète et qui stipulerait la marche à suivre lorsqu'une critique est émise. Comme en montagne, une bonne approche de la critique au sein de la clinique peut sauver des vies.

\section{Des analyses qualitatives et quantitatives}

Le développement croissant des unités de soins intermédiaires en Suisse accentue la nécessité d'une facturation précise des prestations fournies dans les U-IMC. Comme Hans Ulrich Rothen de la Commission tarifaire de la SSMI le souligne, une adaptation du catalogue des forfaits par cas (DRG) aux réalités des services des unités de soins intermédiaires est actuellement en cours.
Il est également important que les U-IMC reconnues en Suisse aient la possibilité d'analyser leurs propres structures et leurs propres processus à tout moment afin d'identifier leurs points forts mais aussi leurs points faibles et d'améliorer ainsi la qualité de la prise en charge des patients à risque, a ajouté Jolanda Contartese, présidente de la CRUIMC de Baden, dans son intervention lors du symposium. En collaboration avec la Commission Données de la Société Suisse de

Dans les unités IMC, les questions d'ordre éthique jouent un rôle très important en raison de l'état généralement critique des patients.

Médecine Intensive (SSMI), un ensemble de données pour les soins intermédiaires (le fameux MDSimc) a par conséquent été rapproché des données minimales de la SSMI (MDSi) introduites il y a quinze ans déjà, et mis en place. Ce MDSimc définit et collecte certains indicateurs clés d'une unité de soins intermédiaires: combien de temps dure en moyenne un séjour en U-IMC? Combien de médecins ou d'infirmières/infirmiers sont affectés à un patient à risque? Quel est le pourcentage de patients admis pour la deuxième fois en U-IMC? Voilà quelques exemples d'indicateurs issus de l'ensemble de données actualisé en permanence et

Il est essentiel de promouvoir une culture ouverte du dialogue permettant la critique envers la hiérarchie et au sein de l'équipe.

qui sera implémenté dans le courant de l'année dans les U-IMC. La collecte de ces données constitue le point de départ des analyses qualitatives et quantitatives des unités IMC en Suisse. Les neuf sociétés spécialisées représentées au sein de la CRUIMC sont responsables de ces données, qui sont systématiquement anonymisées.

Vous trouverez de plus amples informations sur les unités de soins intermédiaires en Suisse sur le site de la CRUIMC: www.swiss-imc.ch.

Crédits photo

Luca Lavina 